\title{
HYPERPYREXIA AND DEATH ASSOCIATED WITH GENERAL ANAESTHESIA
}

\author{
L. E. DavIES, M.D., AND H. B. Graves, B.A., M.D., F.R.C.P.(c)
}

A TWENTY-FOUR-YEAR-OLD FEMALE PATIENT whose only symptoms were related to gall bladder disease was booked for a cholecystectomy and cholangiogram on the morning of October 6, 1964.

She was nicely sedated on secobarbital gr. iss., meperidine $100 \mathrm{mg}$., and hyoscine $0.4 \mathrm{mg}$. Preoperative blood pressure was 120/80. Pulse was 80 and regular. Induction at 7.50 A.M. was uneventful with thiopentone $350 \mathrm{mg}$., and succinylcholine $50 \mathrm{mg}$. The patient was intubated with a number 8 Portex tube with cuff inflated.

Anaesthesia was maintained with $\mathrm{N}_{2} \mathrm{O}+\mathrm{O}_{2}+$ halothane. When respirations returned, relaxation was obtained with gallamine $120 \mathrm{mg}$. Respirations were manually controlled throughout. Blood pressure was steady at $120 / 80$, pulse remained rapid at $120 / \mathrm{min}$. following gallamine. Further gallamine $40 \mathrm{mg}$. was needed to close the peritoneum at 9.45 A.M. About 10.15 the pulse rate gradually increased to 160 . Ventilation became difficult, and the patient was a little off colour. The anaesthetic bag and cannister were observed to be very warm. Only oxygen was given, and jerky spontaneous respirations became apparent. The patient's skin was very warm.

Closure was rapidly completed at 10.30 A.M. The patient did not rouse, respirations remained jerky, there was marked rigidity of the limbs, and peripheral cyanosis. The pulse gradually slowed and the pressure fell. Rectal temperature was $104^{\circ} \mathrm{F}$.

The Head of the Department of Neurosurgery examined the patient and concluded that she had suffered major damage to her brain stem and that no therapy was indicated. The rectal temperature rose to $108^{\circ}$ even after tepid and alcohol sponging at 10.45 A.M.

At 11.00 A.M. the patient was moved to the post-anaesthetic recovery room in bed. Respirations were assisted, but she gradually deteriorated, and died at 11.27 A.M.

Previous history. Cholelithiasis with jaundice 3 years' duration. Loss of weight: 20 lbs. in 3 months. Married, 3 daughters.

Laboratory investigations. Prothrombin 90 per cent, hgb. $13.6 \mathrm{gm}$. (94 per cent), W.B.C. 6000 , sed. rate $21 \mathrm{~mm} . / \mathrm{hr}$., chloride $102 \mathrm{mEq}$., bicarbonate $32 \mathrm{mEq}$., sodium $138 \mathrm{mEq}$., potassium $4.5 \mathrm{mEq}$, urea $\mathrm{N} 11 \mathrm{mg}$. per cent, urinalysis negative.

Physical examination. Negative except for biliary tract disease.

An autopsy was done which included careful microscopic examination of sections of brain. The pathologist's final diagnosis was: acute pulmonary oedema, acute cerebral oedema, probable idiosyncrasy to anaesthetic agent. 


\section{RésuMé}

Il s'agit d'une jeune femme de 24 ans dont les seuls symptômes se rattachaient à un trouble de la vésicule biliaire que l'on a cédulée pour cholécystectomie et choliangiographie le matin du 6 octobre 1964.

Elle avait reçue une bonne sédation: du sécobarbital, de la mépéridine $100 \mathrm{mg}$. et de l'hyoscine $0.4 \mathrm{mg}$. La tension artérielle préopératoire était de $120 / 80$, son pouls était régulier à $80 \mathrm{par} \mathrm{min}$. A $7.50 \mathrm{hres}$ du matin, l'induction a été faite avec $350 \mathrm{mg}$. de thiopentone, elle n'a présenté rien de particulier; pour l'intuber, on a donné $50 \mathrm{mg}$. de succinylcholine et l'on a employé un tube portex No. 8 avec ballonnet que l'on a gonflé.

Le maintien de l'anesthésie s'est fait avec du protoxyde d'azote, de l'oxygène et de l'halothane. Au retour de la respiration, l'on a donné $120 \mathrm{mg}$. de gallamine pour procurer du relachement. Nous avons controlé la respiration à la main durant toute l'anesthésie. La tension artérielle s'est maintenue à 120/80 et le pouls est demeuré rapide à 120 à cause de la gallamine. A 9.45 hres, pour la fermeture du péritoine, nous avons dû ajouter $40 \mathrm{mg}$. de gallamine. Vers 10.15 hres le pouls s'est accéléré graduellement jusqu’à 160. La ventilation est devenue difficile et la malade, moins bien colorée. L'on a donné de l'oxygène pur et une respiration spontanée saccadée est apparue. La peau de la malade était très chaude.

L'on a fermé rapidement à 10.30 hres du matin. La malade ne s'est pas réveillée, la respiration est demeurée saccadée, il existait une rigidité marquée des membres et de la cyanose périphérique. Le pouls s'est ralenti, la pression a tombé, la température rectale était de $104^{\circ} \mathrm{F}$.

Le directeur du département de neurochirurgie a examiné la malade, a déclaré qu'elle avait des dommages cérébraux étendus et qu'il n'y avait pas lieu d'instituer de thérapeutique. La température rectale a atteint $108^{\circ} \mathrm{F}$. même après des bains d'éponges à l'alcool a 10.45 A.M.

A 11.00 on a transporté la malade dans son lit a la salle de réveil. On a assisté sa respiration, mais son état général s'est détérioré graduellement et, a 11.27 A.M., elle mourait.

Histoire antérieure: Cholélithiase et jaunisse durant 3 ans. Perte de poids: $20 \mathrm{lbs}$ en trois mois. Mariée, mère de 3 filles.

Analyses de laboratoire: Prothrombine 90\%, Hb $13.6 \mathrm{gm}$. 94\%, G.B. 6000, sédimentation $21 \mathrm{~mm} . / \mathrm{hr}$, chlorures $102 \mathrm{mEq}$, bicarbonates $32 \mathrm{mEq}$, sodium $138 \mathrm{mEq}$, potassium $4.5 \mathrm{mEq}$., urée $11 \mathrm{mg} . \%$, urine: rien à noter.

Examen physique: Rien a noter à part les troubles vésiculaires.

Une autopsie a été pratiquée y compris un examen microscopique soigneux de certaines sections du cerveau. Le diagnostic final du pathologiste: œdème pulmonaire aigu, œdème cérébral aigu, idiosyncrasie probable aux agents anesthésiques. 